\title{
Review
}

\section{Review article on COVID-19 and Guillain-Barré syndrome}

\author{
U. Juhi Patnaik ${ }^{1, *}$ \\ ${ }^{1}$ Jawaharlal Nehru Medical College, Datta Meghe Institute of Medical Sciences, 442001 Sawangi Meghe, Wardha
}

\section{TABLE OF CONTENTS}

\section{Abstract \\ 2. Introduction \\ 3. Methods \\ 4. Discussion}

\author{
4.1 COVID-19 \\ 4.2 Pathophysiology of COVID-19 \\ 4.3 Neurological pathophysiology of COVID-19 and Guillain-Barré syndrome \\ 4.4 Variants of Guillain-Barré syndrome \\ 4.5 Treatment of COVID-19 \\ 4.6 Diagnosis of Guillain-Barré syndrome \\ 4.7 Treatment protocol in Guillain-Barré syndrome
}

5. Conclusions

6. Author contributions

7. Ethics approval and consent to participate

8. Acknowledgment

9. Funding

10. Conflict of interest

11. References

\section{Abstract}

The tale COVID infection pandemic or as far as we might be concerned better, COVID-19, has assaulted society on a worldwide scale. For the unenlightened, the sickness is brought about by the specific infection Severe Acute Respiratory Syndrome Coronavirus 2 (SARS-CoV2). It is only from time to time that we have a pandemic seething on that has carried with itself a particularly humongous size of harm and on each and every front of the human culture, be it clinical, practical, social or pretty much anything. Theemerging coronavirus disease 2019 (COVID-19) has neurological symptoms comparable to that of the Extreme Acute Respiratory Syndrome Coronavirus (SARSCoV) and MERS-CoV. Medical symptoms such as pain in head, vomiting, nausea, dizziness, muscle pain, anosmia, ageusia, and disorder of consciousness are present in COVID-19 affected people. These signs confirm that the COVID-19 infection affects the nervous system. But nerve affecting manifestations of COVID-19 infection are underreported. Guillain-Barré Syndrome (GBS) is a condition that often arises in various forms. According to the evaluation case reports so far from the start of COVID-19 infection, GBS could be associated with COVID-19 infec-

tion. There was a systematic review and published cases that suggested that a broad age range with male predominance was affected. There were respiratory and/or systemic symptoms in most patients and they developed GBS manifestations after COVID-19. However, asymptomatic cases of COVID-19 have also been identified. The distribution of clinical variants and electrophysiological subtypes is close to that of classical GBS, with a higher prevalence of classical sensorimotor form and acute inflammatory demyelinating polyneuropathy. It seems like it is important to pay attention to the neurological effects of COVID-19.

\section{Introduction}

Coronaviruses (CoV) 2019 are a vast family of viruses that can cause diseases ranging from the common cold to more serious diseases, such as Middle East Respiratory Syndrome (MERS-CoV) and Extreme Acute Respiratory Syndrome (SARS-CoV). Another strain, which has not been recently portrayed in people, is a novel coronavirus $(\mathrm{nCoV})[1]$.

Covids are zoonotic, which implies that they are circulated among creatures and people. Definite exami- 
nations demonstrated that SARS-CoV was sent to people fromcivet felines and MERS-CoV was sent to people from dromedary camels. In creatures which have not yet contaminated people, many known COVID are circling.

Respiratory symptoms, fever, cough, shortness of breath and breathing problems are typical signs of infection. Pneumonia, extreme acute respiratory syndrome, kidney failure and evendeath may be caused by infection in more severe cases. Daily hand washing, covering the mouth and nose while coughing and sneezing, cooking meat and eggs thoroughly are common recommendations to avoid infection from spreading. Avoid direct contact with those with respiratory disease symptoms, such as coughing and sneezing [2].

The nervous system is affected. Due to its effects on the central nervous system (CNS) (headache, dizziness, consciousness disturbance, acute brain disease, seizures, and ataxia), peripheral nervous system (PNS) (anosmia, ageusia, vision impairment, nerve pain), and skeletal muscles, the neurological symptoms of COVID-19 infection are due to its effects on the central nervous system (CNS). It also can cause encephalitis and stroke [3].

Guillain-Barré Syndrome (GBS) is a peripheral nervous system-related autoimmune condition. It is a rapid progressive, acute, generalized polyradiculoneuropathy which is associated with an infection of Campylobacter jejuni, Epstein-Barr virus, influenza, or cytomegalovirus in most of the cases. Guillain-Barré syndrome represents the most common cause of acute flaccid paralysis [4, 5].

Progressive limb weakening and reduction in or loss of tendon reflexes are the clinical characteristics of Guillain-Barré syndrome (hyporeflexia and areflexia, respectively). Protein concentrations in the cerebrospinal fluid (CSF) increase in this condition, while the number of white cells is normal. A viral or bacterial infection normally triggers GBS. The immune system is stimulated in response to the antigen and the nerve roots and peripheral nerves are injured because of this antigen's structural resemblance to axons and myelin. The clinical variants of GBS includes the classical sensory motor type, Miller Fisher Syndrome (MFS), bilateral paraesthesia facial palsy, pure motor, pure sensory, paraparetic, pharyngeal-cervical-brachial forms, polyneuritis cranialis (Guillain-Barré syndromeMiller Fisher Syndrome overlap) and Bickerstaff brainstem encephalitis.

As regards electrophysiological characteristics, three major subtypes are identified: AIDP, acute motor axonal neuropathy (AMAN) and acute motor axonal neuropathy (AMSAN). Guillain-Barré syndrome associated with COVID-19 is now widely reported, but the strength and mechanism of the association and the clinical and electrodiagnostic patterns remain unclear.

\section{Methods}

All types of published reportswere analyzed that are on COVID-19-associated Guillain-Barré syndrome. A search was performed on PubMed, Google Scholar databases and individual case reports and some case series, and cohort studies were identified. Full texts articles were acquired from the Google Scholar and PubMed. For COVID-19 test that has to be done to screen RT PCR (Reverse Transcriptase Polymerase Chain Reaction), serological tests, blood tests, chest x ray, HRCT (High Resolution CT) were performed in case studies. For GuillainBarré syndrome, electromyography, serology, immunological studies, CSF studies had been done in case reports. Neuro imagining studies have been also analyzed, clinical variants and presentations have also been seen and identified by electromyography.

\section{Discussion}

\subsection{COVID-19}

COVID-19 is caused by SARS-CoV-2 (Severe Acute Respiratory Syndrome Coronavirus 2) a beta coronavirus single stranded RNA virus, it is called as it is genetically similar to SARS coronavirus outbreaks in 2002 [2]. Coronavirus are a family of virus affecting humans and many species of animals like bats, cats and others. It commonly affects the upper respiratory tract causing common cold. The coronavirus affecting animals can evolve and affect the human example includes SARS (Severe Acute Respiratory Syndrome Coronavirus) and the Middle East Respiratory Syndrome (MARS). In December 2019, Wuhan in Hubei, China reported many cases of unusual cases of pneumonia. In January, scientists identified the source of the infections as "novel coronavirus". The same cases had started being detected in other countries like Thailand, USA, Europe. On 30 January 2020, WHO declared this outbreak of SARS-CoV-2 as a global public emergency outbreak. On 11 March 2020, COVID-19 was declared a global pandemic $[3,6]$. The pandemic which has arised was initially an arcane to this world. The world has been cajoled by this leading information which has not yet been proved if china has really released this virus intentionally or accidently. But this situation shouldn't be circumspected as it now has lead to wide range of death and left trauma to the living families. There has been many kinds of rumors regarding the origination of this infection, starting from china using it as a war to its accidental contamination of the virus by an intern in the lab leading to being first affecting the people of the animal market who eat different kinds of animals like bat, etc. Initially the china killed the scientist who discovered this virus and tried to warn and tell the people of that country but he had been silenced. Later the people started killing all of their pets and animals of street to get rid of the infection contaminating the society. Later other countries 
like Korea also start slaughtering the people down who got infected with this. COVID has taken the mind of people and their lives as well as the lives of animals and all age groups have succumbed to this pandemic.

\subsection{Pathophysiology of COVID-19}

The virus enters the human body via inhalation by nose and then it enters the nasal cavity. Binding of SARS$\mathrm{CoV}-2$ to the ciliated secretory cells in the nasal epithelium via ACE2. The viral replication takes place and there is limited immune response to virus as the viral load is high. The conducting airways, upper respiratory tract and lower respiratory tract starts getting involved. Then the invasion and infection of type 2 pulmonary alveolar epithelial cells occurs via ACE2 by viruses. There are two ways in which the immune response will take place after affecting type 2 pulmonary alveolar epithelial cells.

The first type of immune response is the Cytokine storm which is the release of IL-1, IL-6, IL-8, IL-10, IL12 , TNF $\alpha$, IFN- $\lambda$ and IFN- $\beta$, CXCL-10, G-CSF, GMCSF, MCP-1 and MIP- $1 \alpha$ which leads to attraction of neutrophils, CD4, CD8 cells along with B cell differentiation. Invasion of all these cells into lung tissues lead to cytotoxicity and lung injury which can lead to cytotoxicity and lung injury which at the final stage can cause alveolar damage with ARDS (Acute Respiratory Distress Syndrome).

The second type of immune response is viral replication and release of viral particles which results in apoptosis of host cells. The virus keeps multiplying and infecting the healthy alveolar epithelial cells with loss of type 1 and type 2 pneumocytes causing ARDS and alveolar damage leading to respiratory failure and end stage is the death $[6,7]$. The whole pathophysiology of COVID-19 infection has been explained in Fig. 1 given below.

4.3 Neurological pathophysiology of COVID-19 and Guillain-Barré syndrome

The neurological mechanism of COVID-19 are many. Via the olfactory neurons, hematogenous spread, the virus goes to ACE2 protein on cells in CNS. Cytokine storm and breakdown of blood brain barrier also occurs when the receptor of the virus binds to ACE2. The neurological sequelae of COVID are dizziness, headache, altered mental status, stroke, ataxia, seizure, anosmia, visual changes, nerve pain, skeletal muscle injury associated with sepsis and multiorgan injury.

Guillain-Barré syndrome is a syndrome that is a rapidly progressive immune theory mediated polyradiculoneuropathy. It is usually in association with preceding infections. Most of the patients have symptoms of infection in preceding 3-6 weeks. Campylobacter jejuni is the likely the most common infection causing Guillain-Barré syndrome. There are many variants of Guillain-Barré syndrome that are, AIDP (Acute Inflammatory Demyelinating Polyneuropathy), AMAN (Acute Motor Axonal Neuropathy), AMSAN (Acute Motor Sensory Axonal Neuropathy),
MF syndrome, Prototypic Bickerstaff encephalopathy. Reports of Guillain-Barré syndrome in patients with COVID19 have been emerging. The first cases have beenreported in Italy after COVID-19 infection. In most of the cases of Guillain-Barré syndrome patients came with presenting features of lower extremity involving weakness and paresthesias. Patients started developing the symptoms during 5-10 days after getting COVID symptoms. Most of the patients developed AMAN which presents as weakness in all four limbs and AIDP variant of Guillain-Barré syndrome which presents as weakness and motor as well as sensory loss. The patients presents with fever, cough, dyspnea, anosmia, ageusia, diarrhea. The time period of onset of GuillainBarré syndrome following COVID infection has been reported approximately 3-24 days. The neurological presentation of Guillain-Barré syndrome varies from sensorimotor, pure motor, MFS, pharyngeal variant. Electromyography subtypes showed demyelinating and axonal subtype. Some of the patients have also succumbed to respiratory failure. The pathophysiology remains unknown and yet to be explained [7-9].

\subsection{Variants of Guillain-Barré syndrome}

There are four of kinds of Guillain-Barré syndrome which presents in different kinds of forms. First being AIDP (Acute inflammatory Demyelinating Polyneuropathy). The inflammatorydemyelinating is suspected to start at level of the nerve roots, leading to electrophysiological conduction slowing with conduction blocks, with extreme muscle weakness. Remyelination can occur at peripheral nerves. It is mainly the most common subtype of this type of syndrome that occurs.

The second variant is Acute Motor Sensory Axonal Neuropathy (AMSAN), which is the most severe form of AMAN (Acute Motor Axonal Neuropathy). Sensory with motor neuron fibers are most likely that is affected with axon degeneration, which cause mainly delayed and incomplete recovery which can be reversible or irreversible. Clinically resembles Acute Motor Axonal Neuropathy but with more sensory symptoms.

The third is Miller Fisher Syndrome (MFS), that is typically presented as ataxia, problem with movement of eye and areflexia. Antibodies that is against GQ1b (a ganglioside component of nerve) are present in most of the patient of this variant. Anti-GQ1b ganglioside commonly is an antigenic target that is not proportionally evident in the motor nerves which definitely innervate extraocular muscles.

The last variant is AMAN (Acute Motor Axonal Neuropathy), which is distinguished from Acute inflammatory Demyelinating Polyneuropathy by its selective involvement of motor nerves and by electrophysiologic pattern of axonal involvement $[10,11]$. 


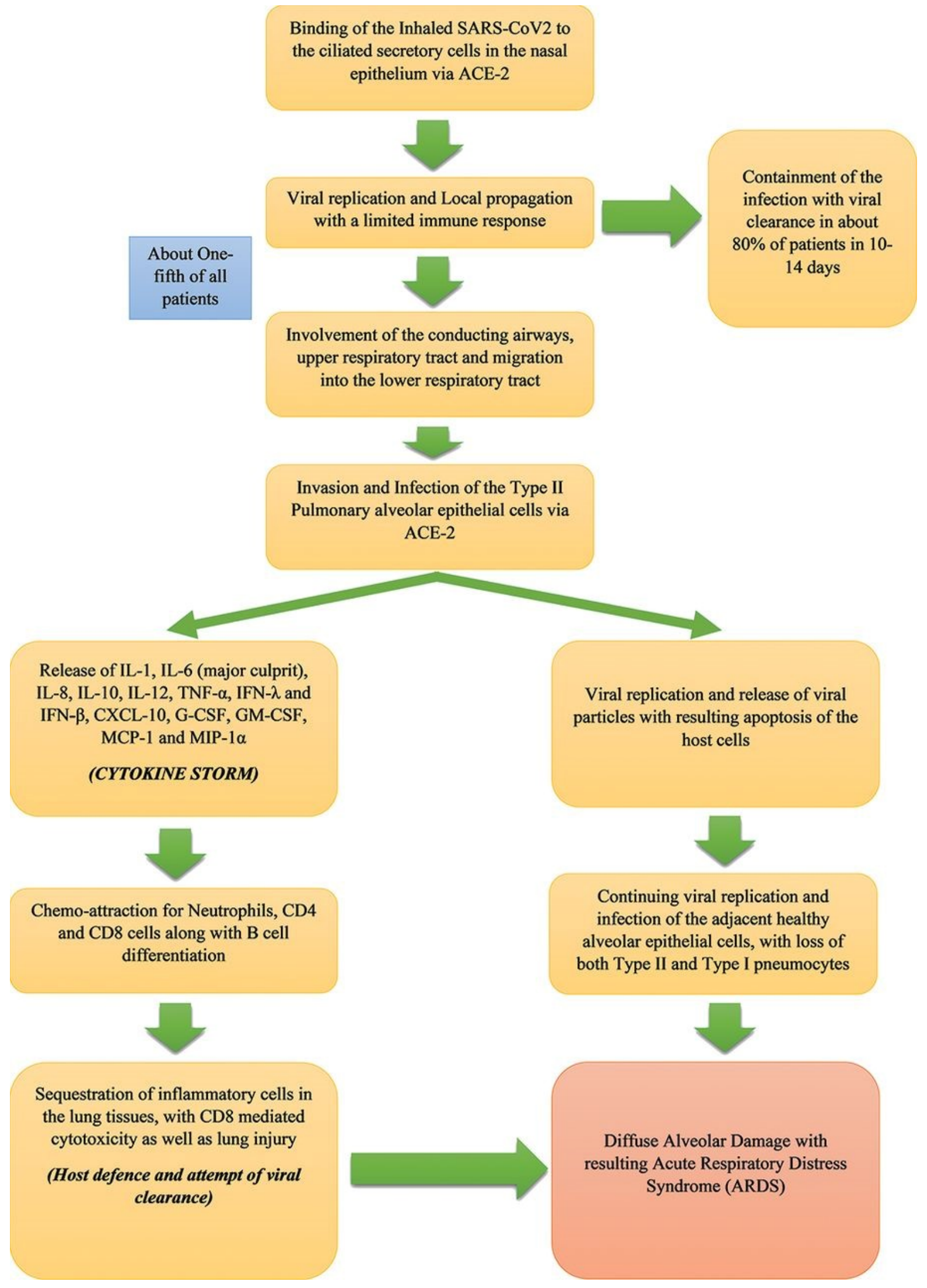

Fig. 1. Pathophysiology of COVID-19. 


\subsection{Treatment of COVID-19}

Treating the COVID-19 with Guillain-Barré syndrome is independently yet simultaneously done.

Treatment of COVID-19-it depends on, if the symptom is mild, moderate, or severe disease.

Mild disease-home isolation is done as soon as the patient is tested positive. Symptomatic management is done. Tab HCQ used to be given. Now prophylactic antibiotics, vitamin $\mathrm{C}$, multi vitamin, zinc, faviparavir, ivermectin, etc. is given to decrease the viral load though it is not the actual treatment.

Moderate disease-hospital admission is required. Oxygen therapy, Iv Methyl prednisolone, prophylaxis with LMWH (enoxaparin), prophylactic broad spectrum antibiotics.

Severe disease- hospital admission, oxygen therapy, ICU transfer, broad spectrum antibiotics, fluids, Remdesivir, Iv Methyl prednisolone, prophylaxis with LMWH (enoxaparin) and ARDS management.

If no improvement occurs then Tocilizumab and convalescent.

Remdesivir is a FDA approved antiviral drug used here. Mostly used in adults and grown up children having good healthy weight.

Favipiravir and ivermectin course may decrease the time duration of illness [12-14].
Precautions to be taken by wearing masks and maintaining social distancing.

Presently the government has released two vaccines against this infection covaxin and covisheild which is some side effects like fever, pain in whole hand, etc.

New drugs have been generated now to fight this virus, barcinitib (Olumiant) which is usually given in rheumatoid arthritis

Barcinitib in combination of that with remdesivir which is usually used in emergency though there is no evidence to support the use of this combination together.

Dexamethasone has been used in very ill patients since the beginning of this pandemic. This makes a scientific sense for the patients who have eventually developed a hyper immune response to viral infection $[15,16]$.

Do not mislead with the treatment protocols of the upcoming drugs for the treatment as it can be harmful to the population. The approved drugs should be given only to avoid the toll of death of the people from this pandemic. Let's hope for the best possibility of the cure and decreased rates of death and increased rate of mortality from the given situation presently.

The patients having this infection cannot donate their organs as they are infected and can infect the live human.

\section{Brighton Diagnostic Criteria for GBS Level of Diagnostic Certainty}

\begin{tabular}{|c|c|c|c|c|}
\hline Symptoms & 1 & 2 & 3 & 4 \\
\hline Bilateral and flaccid weakness of limbs & + & + & + & $+/-$ \\
\hline $\begin{array}{l}\text { Decreased or absent deep tendon } \\
\text { reflexes in weak limbs }\end{array}$ & + & + & + & $+/-$ \\
\hline $\begin{array}{l}\text { Monophasic course and time between } \\
\text { onset-nadir }=12 \text { hours to } 28 \text { days }\end{array}$ & + & + & + & $+/-$ \\
\hline $\begin{array}{l}\text { Absence of alternative diagnosis for } \\
\text { weakness }\end{array}$ & + & + & + & $+/-$ \\
\hline CSF cell count $<50 / \mathrm{ml}$ & + & $+/-^{a}$ & - & $+/-$ \\
\hline CSF protein concentration $>60 \mathrm{mg} / \mathrm{dL}$ & + & $+/-^{a}$ & - & $+/-$ \\
\hline $\begin{array}{l}\text { Nerve conduction study findings } \\
\text { consistent with one of } \\
\text { the subtypes of GBS }\end{array}$ & + & $+/-^{a}$ & - & $+/-$ \\
\hline
\end{tabular}

Fig. 2. Brighton diagnostic criteria for GBS. 


\subsection{Diagnosis of Guillain-Barré syndrome}

Suspecting Guillain-Barré syndrome includes the clinical presentation which includes:

Rapid increasing and progressive bilateral limb weakness with or without sensory deficits, hyporeflex or areflexia, facial or bulbar palsy, ophthalmoplegia and problem in gait. The diagnosis includes-checking the diagnostic criteria, exclude the other causes of similar neurological manifestations. Routine tests in laboratory, Cerebrospinal fluidtests, electrophysiological studies should be done to diagnose the syndrome and its kinds. In spinal fluid increased number of mononuclear cells or polymorphonuclear cells (>50 cells per $\mu \mathrm{L}$ ). There is Brighton Diagnostic criteria for GBS which is mentioned below in Fig. 2 for diagnosing according to the clinical presentation of the patient [17-19].

\subsection{Treatment protocol in Guillain-Barré syndrome}

\section{Acute care includes:}

Identifying the clinical presentation and symptoms, to admit the patient should have one or more of the following symptoms:

Rapidly progression of weakness;

Severe autonomic non-functioning or dysphagia; Progressive respiratory distress;

EGRIS (Erasmus GBS Respiratory Insufficiency score) $>4$.

The management of the patient with GBS should be started when he is not able to walk $>10 \mathrm{~m}$, rapid progression of weakness, severe autonomic or dysphagia, respiratory distress.

Monitoring is very crucial in this situation to avoid permanent disability of the patient, regular assessment of the muscle strength, lung functioning tests, swallowing function should be done. Also access the autonomic functions like blood pressure, heart rate, bladder and bowel control.

To manage respiratory failure, mechanical ventilation is required, percutaneous dilatational tracheostomy is the last resort to be done if other treatment fails.

DVT prophylaxis should be given since the patients are mostly bed ridden. Heparin and support stockings are given, oral anticoagulant and warfarin is given. as possible.

Nasogastric tube feeding should be started as soon

Immunotherapy is the treatment of choice which includes plasma exchange and IVIg (immunoglobulin) [19, 20].

Plasma exchange should be done as 200-250 $\mathrm{mL} / \mathrm{kg}$ for 5 sessions.

Plasmapheresis, Steroids are also effective. IVIg is the most effective. It is given in $0.4 \mathrm{~g} / \mathrm{kg}$ as per body weight every day for 5 days.

IVIg and plasma exchange has very good prognosis in patients of GBS of all variants.
The patient should also be monitored to avoid early complications like- choking, constipation, cardiac arrhythmias, corneal ulceration, infections, dietary deficiency, deep vein thrombosis, hyponatraemia, pain, pressure ulcers, delirium, compression neuropathy, depression, limb contractures, urinary retention.

Long term care of Guillain-Barré syndrome patient includes:

Predicting the outcome of the patient by calculating Megos (Modified Erasmus GBS Respiratory Insufficiency score) on admission, recovery can take for more than 3 years after onset.

Rehabilitation is an important part of the treatment for those who are disabled due to Guillain-Barré syndrome. The rehabilitation is important to be started as early as possible. Management of long term complaints like pain, weakness and psychological distress should be done. GBS patient organizations are also present all around the world, which will be beneficial for the patients [12, 21, 22]. Interesting studies on Guillain Barre syndrome were reported by Yadav et al. [23] and Balwani et al. [24]. Cases of myeloneuropathy and polyneuropathy were reported by Jain et al. [25] and Acharya et al. [26]. Rathi et al. [27] reported on nerve conduction studies of peripheral motor and sensory nerves in the subjects with prediabetes. A few of the related studies on COVID were reported [28-30].

\section{Conclusions}

As there are less number of cases which have been reported of GBS and COVID-19, the pathophysiology is yet to be explained of the relation between the GBS with COVID-19 and remains unknown. There is increase in occurrence of Guillain-Barré syndrome after COVID-19 infection (SARS-CoV-2). The average time of all COVID-19 positive patients to develop Guillain-Barré syndrome is $<$ 2 weeks on an average as per the case reports. Patients presenting with an acute paralytic disease like Guillain-Barré syndrome, encephalitis or myositis should be evaluated for Guillain-Barré syndrome. Ansomnia, ageusia, cranial neuropathies and lymphocytopenia are the red flags. The EMG obtained of the affected patients have shown the different variants of Guillain-Barré syndrome. Those patients have been treated with IVIg and some with plasma exchange and have shown good prognosis and recovery of all types of Guillain-Barré syndrome variants. Some have died due to respiratory failure. The COVID-19 infection should be treated as per the guidelines and protocols. Biomarkers are used for diagnosing Guillain-Barré syndrome. GuillainBarré syndrome should also be simultaneously treated as per the treatment guidelines of Guillain-Barré syndrome. After reviewing the articles and case studies on this topic, the pathophysiology still remains unknown and hence future studies should be done like testing bio marker for serology SARS-CoV-2 and case control study to determine as- 
sociation between COVID-19 infection and GBS and immunological studies should also be done to find out the relationship between these two diseases. Early diagnosis, monitoring and treatment is very necessary for good prognosis. Keeping in mind all the information that has been given, this review article shows the significant relation between both diseases which is yet to find out. With some diligence and work the world will be free from this pandemic.

\section{Author contributions}

Author conducted the literature search, sorted and compiled the relevant information and prepared the manuscript for final publication.

\section{Ethics approval and consent to participate}

Not Applicable.

\section{Acknowledgment}

I acknowledge the support of Dr. S.Z. Quazi and Dr. Manoj Patil from Research and Development, DMIMS, Wardha in preparing this review article and final formatting for publication.

\section{Funding}

Datta Meghe Institute of Medical Sciences, Wardha, India.

\section{Conflict of interest}

The author declare no conflict of interest.

\section{References}

[1] Fauci AS, Lane HC, Redfield RR. Covid-19 - navigating the uncharted. New England Journal of Medicine. 2020; 382: 12681269.

[2] Velavan TP, Meyer CG. The COVID-19 epidemic. Tropical Medicine \& International Health. 2020; 25: 278-280.

[3] Shereen MA, Khan S, Kazmi A, Bashir N, Siddique R. COVID19 infection: origin, transmission, and characteristics of human coronaviruses. Journal of Advanced Research. 2020; 16: 91-98.

[4] Parasher A. COVID-19: current understanding of its pathophysiology, clinical presentation and treatment. Postgraduate Medical Journal. 2020. (in press)

[5] Caress JB, Castoro RJ, Simmons Z, Scelsa SN, Lewis RA, Ahlawat A, et al. COVID-19-associated Guillain-Barré syndrome: the early pandemic experience. Muscle and Nerve. 2020; 62: 485-491.

[6] Pithadia AB, Kakadia N. Guillain-Barré Syndrome (GBS). Pharmacological Reports. 2010; 62: 220-232.

[7] Hutchins KL, Jansen JH, Comer AD, Scheer RV, Zahn GS, Capps AE, et al. COVID-19-Associated bifacial weakness with paresthesia subtype of Guillain-Barré syndrome. American Journal of Neuroradiology. 2020; 41: 1707-1711.
[8] Webb S, Wallace VC, Martin-Lopez D, Yogarajah M. GuillainBarré syndrome following COVID-19: a newly emerging postinfectious complication. BMJ Case Reports. 2020; 13: e236182.

[9] Ellul MA, Benjamin L, Singh B, Lant S, Michael BD, Easton A, et al. Neurological associations of COVID-19. The Lancet Neurology. 2020; 24: 767-783.

[10] Rahimi K. Guillain-Barre syndrome during COVID-19 pandemic: an overview of the reports. Neurological Sciences. 2020; 41: 3149-3156.

[11] Martín-Aguilar L, Camps-Renom P, Lleixà C, Pascual-Goñi E, Díaz-Manera J, Rojas-García R, et al. Serum neurofilament light chain predicts long-term prognosis in Guillain-Barré syndrome patients. Journal of Neurology, Neurosurgery \& Psychiatry. 2021; 92: 70-77.

[12] Meena AK, Khadilkar SV, Murthy JMK. Treatment guidelines for Guillain-Barré syndrome. Annals of Indian Academy of Neurology. 2011; 14: S73-S81.

[13] Kory P, Meduri GU, Iglesias J, Varon J, Marik PE. Clinical and scientific rationale for the "MATH+" hospital treatment protocol for COVID-19. Journal of Intensive Care Medicine. 2021; 36: 135-156.

[14] Cascella M, Rajnik M, Cuomo A, Dulebohn SC, Di Napoli R. Features, evaluation, and treatment of coronavirus (COVID-19). US: StatPearls Publishing. 2021.

[15] Abrams RMC, Kim BD, Markantone DM, Reilly K, PanizMondolfi AE, Gitman MR, et al. Severe rapidly progressive Guillain-Barré syndrome in the setting of acute COVID-19 disease. Journal of NeuroVirology. 2020; 26: 797-799.

[16] Zito A, Alfonsi E, Franciotta D, Todisco M, Gastaldi M, Cotta Ramusino M, et al. COVID-19 and Guillain-Barré syndrome: a case report and review of literature. Frontiers in Neurology. 2020; 11: 909-909.

[17] Camdessanche J-P, Morel J, Pozzetto B, Paul S, Tholance Y, Botelho-Nevers E. COVID-19 may induce Guillain-Barré syndrome. Revue Neurologique. 2020; 176: 516-518.

[18] Parasher A. COVID-19: current understanding of its pathophysiology, clinical presentation and treatment. Postgraduate Medical Journal. 2020. (in press)

[19] Fokke C, van den Berg B, Drenthen J, Walgaard C, van Doorn PA, Jacobs BC. Diagnosis of Guillain-Barré syndrome and validation of Brighton criteria. Brain. 2014; 137: 33-43.

[20] Dalakas MC. Guillain-Barré syndrome: the first documented COVID-19-triggered autoimmune neurologic disease. Neurology - Neuroimmunology Neuroinflammation. 2020; 7: e781.

[21] Manganotti P, Bellavita G, D’Acunto L, Tommasini V, Fabris M, Sartori A, et al. Clinical neurophysiology and cerebrospinal liquor analysis to detect Guillain-Barré syndrome and polyneuritis cranialis in COVID 19 patients: a case series. Journal of Medical Virology. 2021; 93: 766-774.

[22] Winer JB. Treatment of Guillain-Barré syndrome. Monthly Journal of the Association of Physicians. 2002; 95: 717-721.

[23] Chaudhary R, Yadav P, Dhaka S, Damke S, Lohiya S. A rare case report of Guillain-Barré syndrome presenting as unilateral facial palsy with isolated acute bulbar palsy. Journal of Pediatric Neurosciences. 2020; 15: 157-159.

[24] Balwani M, Bawankule C, Ramteke V, Pasari A. Hepatitis C virus, directly acting antivirals and Guillain-Barré syndrome. Saudi Journal of Kidney Diseases and Transplantation. 2018; 29: 1237-1239.

[25] Jain J, Banait S, Tiewsoh I, Choudhari M. Kikuchi's disease (histiocytic necrotizing lymphadenitis): a rare presentation with acute kidney injury, peripheral neuropathy, and aseptic meningitis with cutaneous involvement. Indian Journal of Pathology \& Microbiology. 2018; 61: 113-115.

[26] Acharya S, Lahole S, Shukla S, Mishra P, Aradhey P. Copper deficiency myeloneuropathy with bicytopenia-a rare case report. International Journal of Nutrition, Pharmacology, Neurological Diseases. 2020; 10: 154-156. 
[27] Rathi N, Taksande B, Kumar S. Nerve conduction studies of peripheral motor and sensory nerves in the subjects with prediabetes. Journal of Endocrinology and Metabolism. 2019; 9: 147150.

[28] Shah P, Naqvi W. Fighting and chasing the rogue virus-Covid19. International Journal of Research in Pharmaceutical Sciences. 2020; 11: 77-80.

[29] Singh AK, Yeola M, Singh N. A review of convalescent plasma therapy an immediately available therapeutic option for Covid19 in light of the available data. Journal of Datta Meghe Institute of Medical Sciences University. 2020; 15: 149-152.
[30] Agrawal D, Jaiswal P, Goyanka B. Diabetes and Covid-19: a review. International Journal of Research in Pharmaceutical Sciences. 2020; 11: 376-379.

Keywords: COVID-19; Guillain-Barré syndrome; Inflammatory; Polyneuropathy; SARS-CoV; MERS-CoV

Send correspondence to: U. Juhi Patnaik, Jawaharlal Nehru Medical College, Datta Meghe Institute of Medical Sciences, 442001 Sawangi Meghe, Wardha, E-mail: juhipatnaik1997@gmail.com 


\section{Temporary page!}

$\mathrm{ET}_{\mathrm{E}} \mathrm{X}$ was unable to guess the total number of pages correctly. As there was some unprocessed data that should have been added to the final page this extra page has been added to receive it.

If you rerun the document (without altering it) this surplus page will go away, because $\mathrm{HT}_{\mathrm{E}} \mathrm{X}$ now knows how many pages to expect for this document. 